\title{
Guideline-concordant therapy and outcomes in healthcare-associated
} pneumonia

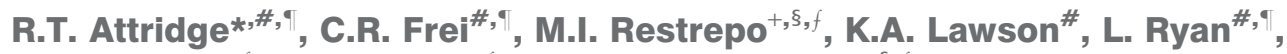 \\ M.J.V. Pugh ${ }^{+, f}$, A. Anzueto ${ }^{+, f}$ and E.M. Mortensen ${ }^{+, \S, f}$
}

ABSTRACT: Healthcare-associated pneumonia (HCAP) guidelines were first proposed in 2005 but have not yet been validated. The objective of this study was to compare 30 -day mortality in HCAP patients treated with either guideline-concordant (GC)-HCAP therapy or GC communityacquired pneumonia (CAP) therapy.

We performed a population-based cohort study of $>150$ hospitals in the US Veterans Health Administration. Patients were included if they had one or more HCAP risk factors and received antibiotic therapy within $48 \mathrm{~h}$ of admission. Critically ill patients were excluded. Independent risk factors for 30-day mortality were determined in a generalised linear mixed-effect model, with admitting hospital as a random effect. Propensity scores for the probability of receiving GC-HCAP therapy were calculated and incorporated into a second logistic regression model.

A total of 15,071 patients met study criteria and received GC-HCAP therapy (8.0\%), GC-CAP therapy $(\mathbf{7 5 . 7 \% )}$ or non-GC therapy $(16.3 \%)$. The strongest predictors of 30 -day mortality were recent hospital admission (OR 2.49, 95\% Cl 2.12-2.94) and GC-HCAP therapy (OR 2.18, 95\% CI 1.86-2.55). GC-HCAP therapy remained an independent risk factor for 30-day mortality (OR 2.12, $95 \% \mathrm{Cl} 1.82-2.48$ ) in the propensity score analysis.

In nonsevere HCAP patients, GC-HCAP therapy is not associated with improved survival compared with GC-CAP therapy.

KEYWORDS: Drug resistance, guidelines for management of pneumonia, health outcomes, pneumonia

n 2005, the American Thoracic Society and the Infectious Diseases Society of America introduced healthcare-associated pneumonia (HCAP) as a new pneumonia classification for patients admitted from the community who have had recent contact with the healthcare system [1]. The presence of an HCAP risk factor at admission (recent hospitalisation, admission from a nursing home/long-term care facility, chronic dialysis, outpatient infusion therapy, home wound care or family member with a multidrug-resistant (MDR) pathogen) indicates a potentially higher risk for an MDR pathogen, and guidelines recommend that HCAP patients receive empirical antibiotic therapy similar to patients with hospital-acquired or ventilator-associated pneumonia.

The HCAP population has been characterised by several recent studies. These data indicate that
HCAP patients are older, present with more severe disease and suffer worse health outcomes than community-acquired pneumonia (CAP) patients [2-6]. Additionally, regional data from the USA suggest higher frequencies of MDR pathogens (specifically Pseudomonas aeruginosa and methicillinresistant Staphylococcus aureus (MRSA)) in HCAP patients $[2,4]$. Although MDR pathogens may be more common in some HCAP cohorts, there are still a significant amount of traditional CAP pathogens isolated in HCAP patients, making HCAP criteria a relatively poor tool for predicting patients with MDR pathogens $[7,8]$. The balance between adequate coverage and overtreatment is difficult, leading to controversy and confusion toward the best approach to caring for these patients.

If treating HCAP patients with guideline-concordant (GC)-HCAP antibiotics demonstrates
AFFILIATIONS

*Feik School of Pharmacy, University of the Incarnate Word,

-Pharmacotherapy Education and Research Center, School of Medicine, The University of Texas Health Science Center at San Antonio,

${ }^{+}$Dept of Medicine, The University of Texas Health Science Center at San Antonio,

sVERDICT Research Program, South Texas Veterans Health Care System, ${ }^{f}$ South Texas Veterans Health Care System, Audie L. Murphy Division San Antonio, and

"College of Pharmacy, The University of Texas at Austin, Austin, TX, USA.

CORRESPONDENCE

C.R. Frei

The University of Texas Health Science Center at San Antonio Pharmacotherapy Education \& Research Center

7703 Floyd Curl Drive

MC 6220

San Antonio

TX 78229-3900

USA

E-mail: freic@uthscsa.edu

Received:

Sept 022010

Accepted after revision:

Feb 262011

First published online:

March 242011 
improved mortality over HCAP patients treated with GC-CAP antibiotics, then the HCAP guidelines are effective and should remain the standard of treatment for HCAP patients. Under this premise, the current study aimed to validate HCAP guidelines by comparing health outcomes in HCAP patients treated with GC-HCAP or GC-CAP therapy.

The primary objective of this study was to compare the effects of GC-HCAP therapy, GC-CAP therapy and non-GC therapy on patient mortality and hospital length of stay (LOS) in a cohort of hospitalised, noncritically ill HCAP patients. The secondary objective was to describe differences in patient mortality and selected bacterial pathogens based on the number of HCAP risk factors present in each patient.

\section{METHODS}

Administrative data from the US Veterans Health Administration (VHA) was used to examine pneumonia care and mortality among patients with HCAP. The VHA databases are repositories of clinical data from more than 150 VHA hospitals and 850 VHA clinics. The Institutional Review Board of The University of Texas Health Science Center at San Antonio (San Antonio, TX, USA) and the South Texas Veterans Health Care System Research and Development committee (San Antonio) approved this study.

\section{Patient eligibility}

All patients were required to have an International Classification of Disease, 9th Edition, Clinical Modification (ICD-9-CM) principal discharge diagnosis of pneumonia (ICD-9-CM codes 480.0483.99 or 485-487) in fiscal years 2002 to 2007 and at least one documented risk factor for HCAP. HCAP risk factors were defined as hospital admission in the previous 90 days, residence in a nursing home in the previous 90 days, receipt of outpatient intravenous antibiotics in the previous 90 days and haemodialysis. Patients undergoing haemodialysis were identified using ICD-9-CM codes (table 1 of online supplementary material). Other HCAP risk factors were obtained from patient records maintained in VHA databases.

Patients were excluded if they were critically ill or did not receive antibiotic therapy within $48 \mathrm{~h}$ of hospital admission. Critically ill patients were excluded in order to minimise differences in level of care between the groups, as critically ill patients suffer increased morbidity and mortality compared with those managed on general medical wards [9]. Patients not receiving antibiotics within $48 \mathrm{~h}$ were excluded in order to reduce potential cases of hospital-acquired pneumonia. Critical illness was identified by: 1) admission to the intensive care unit (ICU) at any time during hospitalisation; 2) the presence of ICD-9-CM codes indicating respiratory organ failure, cardiovascular organ failure or invasive mechanical ventilation; or 3) the receipt of any vasopressor or inotrope. Vasopressors and inotropes included dobutamine, dopamine, epinephrine, isoproterenol, metaraminol, norepinephrine and vasopressin.

\section{Baseline characteristics}

Baseline demographics were recorded at the time of admission and comorbid illnesses were determined using ICD-9-CM codes from outpatient and inpatient care in accordance with the Charlson comorbidity scoring system [10,11]. Patient race was recorded for white and black patients, and ethnicity was reported for patients identifying themselves as Hispanic. Native Americans, Hawaiians and patient records missing racial information were reported as "other." Tobacco use was defined as patients with a diagnosis of nicotine dependence, a recorded visit to a VHA tobacco cessation clinic, a current procedural terminology (CPT) treatment code for smoking (99406 or 99407), or an outpatient prescription for a smoking cessation product (Zyban ${ }^{\circledR}$, varenicline, Nicotrol ${ }^{\circledR}$ or nicotine replacement). ICD-9CM codes were used to identify patients with alcohol abuse/ dependence and organ failure [12-14]. Medication use in the 90 days prior to admission was documented for cardiovascular medications, antidiabetic medications, inhaled corticosteroids, systemic corticosteroids (oral and/or injectable) and pulmonary medications (table 2 of online supplementary material).

\section{Antibiotic therapy and bacterial pathogens}

Antibiotic therapy received within the first $48 \mathrm{~h}$ of admission was evaluated using established consensus guidelines (table 1) [1,15]. Patients receiving additional antibiotics beyond the minimum required to satisfy GC-HCAP or GC-CAP therapy remained in their respective treatment groups. The subset of patients who received both GC-HCAP and GC-CAP therapy was considered to have received GC-HCAP therapy. Patients receiving antibiotics that were not concordant with either CAP or HCAP guidelines were considered to have received non-GC therapy.

Pneumonia pathogens were identified using ICD-9-CM discharge diagnosis codes. Proportions of pneumonia due to Streptococcus pneumoniae, S. aureus and Pseudomonas were compared by the number of HCAP criteria per patient. S. pneumoniae was selected because it is the most common pathogen in CAP and is generally susceptible to guideline-recommended CAP regimens. S. aureus and Pseudomonas were selected because, in patients

TABLE 1 Definitions of community-acquired pneumonia (CAP) and healthcare-associated pneumonia (HCAP) guidelineconcordant (GC) therapy (not intensive care unit patients)

GC-CAP therapy

GC-HCAP therapy

$\beta$-lactam* plus macrolide ; respiratory fluoroquinolone ${ }^{+}$

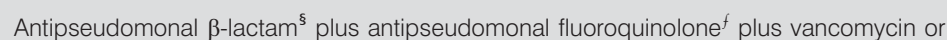
linezolid;

antipseudomonal $\beta$-lactam ${ }^{\S}$ plus aminoglycoside ${ }^{\# \#}$ plus vancomycin or linezolid

\#: includes cefotaxime, ceftriaxone, ampicillin or ertapenem; ${ }^{\uparrow}$ : includes azithromycin, clarithromycin or erythromycin (doxycycline may be substituted); ${ }^{+}$: includes

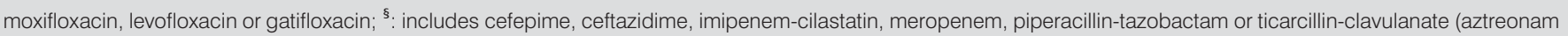
may be substituted in penicillin-allergic patients); ${ }^{f}$ includes ciprofloxacin or levofloxacin; ${ }^{\# \#}$ : includes gentamicin, tobramycin or amikacin. 
without specific risk factors, guideline-recommended CAP therapy has inadequate activity toward MRSA and all Pseudomonas [15]. ICD-9-CM codes used during the study period do not differentiate between methicillin-sensitive $S$. aureus and MRSA; therefore, all $S$. aureus species were included in our analyses. While not reflected in all HCAP data, regional data from the USA suggest MRSA may account for more than half of all $S$. aureus isolates $[2,4]$.

\section{Hospital LOS and mortality}

The primary outcome was 30-day mortality. Previous research has indicated that 30-day mortality is a more accurate measure of pneumonia-related mortality than 90-day mortality [16]. Admission and discharge dates were extracted for each hospital stay and LOS was defined as the date of discharge minus the date of admission plus one day. 30- and 90-day mortality were determined using date of death provided by the VHA status file. This method has a 98\% exact agreement with the gold standard method (the National Death Index) to ascertain mortality [17].

\section{Statistical analysis}

All statistical analyses were conducted using JMP 8.0 ${ }_{\circledR}$ (SAS Corp., Cary, NC, USA) and Stata 10 (StataCorp, College Station, TX, USA). Due to the large sample size, we defined statistical significance as a two-tailed $\alpha \leqslant 0.0001$ for bivariate comparisons. In comparisons among the three treatment groups, GC-HCAP was used as the reference group, and was compared with both the GC-CAP and non-GC groups. In multivariable logistic regression models, a two-tailed $\alpha \leqslant 0.05$ was used.

Patient demographics, baseline characteristics, comorbid conditions, bacterial pathogens and mortality were compared between groups. Dichotomous variables were compared using Chi-squared tests or Fisher's exact tests. All continuous variables were tested for normality with the Shapiro-Wilk W-test and were found to have non-normal distributions; therefore, comparisons were performed with Wilcoxon rank sum tests. Chi-squared and Kruskal-Wallis tests were used to compare differences in mortality and bacterial pathogens by the number of HCAP criteria per patient.

A generalised linear mixed-effect model with admitting hospital as a random effect was used to examine the association between the receipt of GC antibiotics and 30-day mortality. To isolate the effects of GC therapy (GC-HCAP versus GC-CAP), patients who received non-GC therapy were excluded from the model. The dependent variable was 30-day mortality and covariates included: patient sex, race, Hispanic ethnicity, individual HCAP risk factors, individual comorbid conditions, tobacco use, alcohol abuse or dependence, prescription for medications from selected medication classes in the previous 90 days, noninvasive mechanical ventilation, organ failure and guideline-concordant antibiotic therapy. All covariates were considered to be clinically relevant a priori. Because almost all (98.5\%) patients with chronic kidney disease (CKD) as a comorbidity were on dialysis, CKD was excluded from the model to avoid collinearity between variables.

To reduce potential bias in this nonrandomised cohort, a propensity score for the receipt of GC-HCAP versus GC-CAP therapy was calculated using a logistic regression model with GC therapy as the dependent variable and all additional variables in the original model as covariates. GC antibiotic therapy and the propensity scores were included as covariates in a second multivariable logistic regression model with 30-day mortality as the dependent variable.

\section{RESULTS}

\section{Overall population}

Figure 1 provides a flow diagram with detailed information regarding exclusion criteria and the final cohort. Of the 62,682 patients with a principal ICD-9-CM discharge diagnosis code of pneumonia, a cohort of 15,071 met the study inclusion criteria. Patients meeting the inclusion criteria were then stratified by receipt of GC-HCAP $(8.0 \%)$, GC-CAP $(75.7 \%)$ or non-GC therapy $(16.3 \%)$.

Patients were elderly (median age $76 \mathrm{yrs}$ ) and predominantly consisted of white males ( $82.1 \%$ white, $98.3 \%$ males), an innate characteristic of the majority of patients in the VHA [18, 19]. Nearly one-quarter of patients $(22.6 \%)$ had more than one $\mathrm{HCAP}$ risk factor on admission. The most prevalent HCAP risk

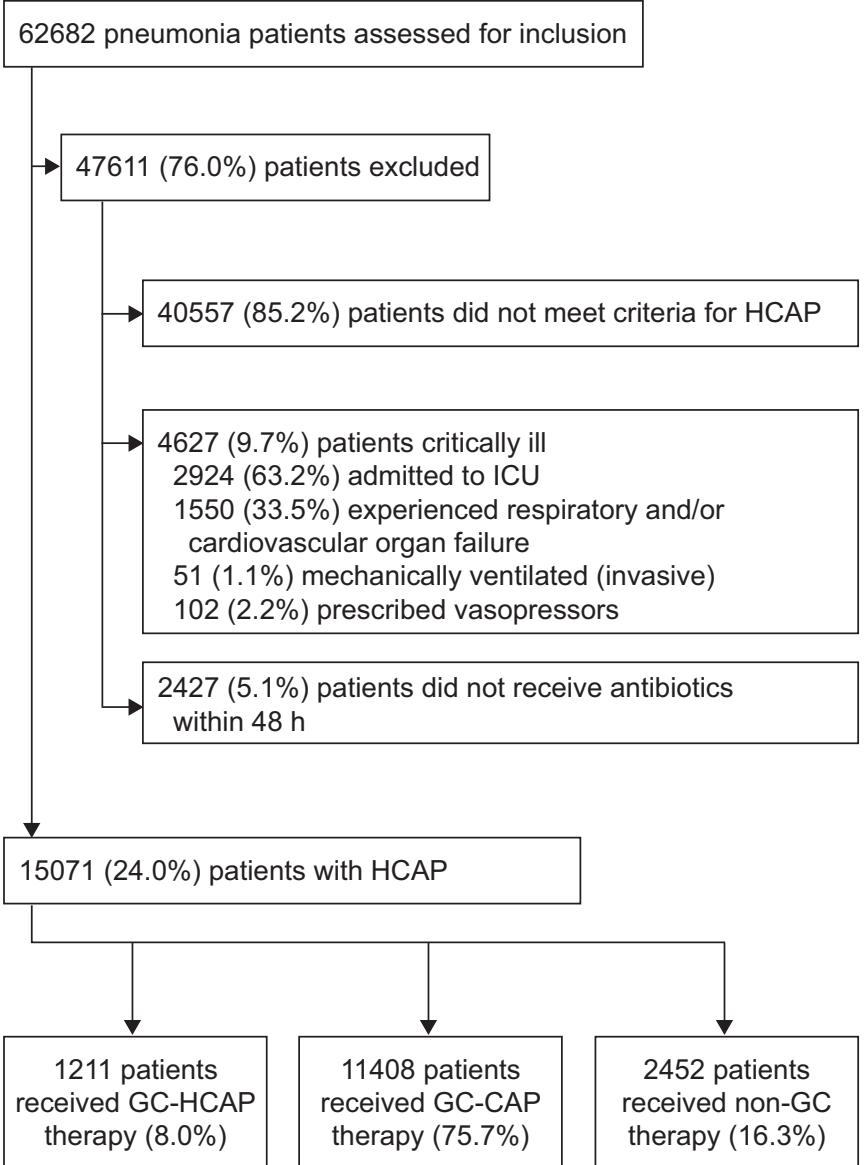

FIGURE 1. Patient inclusion and exclusion flow diagram. HCAP: healthcareassociated pneumonia; ICU: intensive care unit; GC: guideline-concordant; CAP: community-acquired pneumonia. HCAP was defined as prior hospitalisation in the previous 90 days, nursing home admission in the previous 90 days, outpatient intravenous antibiotic therapy in the previous 90 days or chronic kidney disease with haemodialysis. 
factor was hospitalisation in the previous 90 days (66.3\%). The median Charlson Index score was 3 (interquartile range (IQR) 2-5) and common comorbid conditions included chronic obstructive pulmonary disease (COPD; 56.6\%), CKD (44.0\%), diabetes $(38.7 \%)$, heart failure $(36.7 \%)$ and neoplastic disease $(29.0 \%)$. Tobacco use was common $(37.6 \%)$ and cardiovascular medications were the most prescribed medications in the 90 days prior to admission (76.1\%). A small group of patients
(1.2\%) received noninvasive mechanical ventilation during admission. Table 2 lists additional baseline characteristics.

\section{Baseline characteristics by group}

There were no significant differences between the GC-HCAP, GC-CAP and non-GC groups in age, sex or race. Charlson Index scores were similar between GC-HCAP and GC-CAP patients (median (IQR) 4 (2-6) versus 4 (2-5); $\mathrm{p}=0.03)$, and

TABLE 2 Baseline characteristics

\begin{tabular}{|c|c|c|c|c|c|c|}
\hline & \multirow[t]{2}{*}{ Overall } & \multirow[t]{2}{*}{ GC-HCAP } & \multirow[t]{2}{*}{ GC-CAP } & \multirow[t]{2}{*}{ Non-GC } & \multicolumn{2}{|c|}{ p-value } \\
\hline & & & & & GC-HCAP versus GC-CAP & GC-HCAP versus non-GC \\
\hline Patients n & 15071 & 1211 & 11408 & 2452 & & \\
\hline Age yrs & $76(70-80)$ & $76(70-80)$ & $76(70-80)$ & $76(70-81)$ & 0.74 & 0.45 \\
\hline Males & 98.3 & 98.2 & 98.4 & 98.2 & 0.61 & 0.89 \\
\hline Black & 13.4 & 15.5 & 13.3 & 12.6 & & \\
\hline Other & 4.5 & 5.1 & 4.2 & 6.0 & & \\
\hline Hispanic ethnicity & 7.2 & 13.1 & 5.8 & 10.6 & $<0.001$ & 0.02 \\
\hline \multicolumn{7}{|l|}{ HCAP risk factors } \\
\hline Hospitalisation within 90 days & 66.3 & 77.7 & 62.4 & 78.9 & $<0.001$ & 0.42 \\
\hline Nursing home resident within 90 days & 2.9 & 3.0 & 2.8 & 3.5 & 0.71 & 0.36 \\
\hline$\geqslant 2$ HCAP risk factors & 22.6 & 31.1 & 21.0 & 25.7 & $<0.001$ & 0.001 \\
\hline Charlson Index score & $3(2-5)$ & $4(2-6)$ & $4(2-5)$ & $3(2-5)$ & 0.03 & $<0.001$ \\
\hline \multicolumn{7}{|l|}{ Comorbid conditions } \\
\hline Myocardial infarction & 10.3 & 10.0 & 10.2 & 11.3 & 0.83 & 0.25 \\
\hline Heart failure & 36.7 & 31.5 & 37.8 & 34.3 & $<0.001$ & 0.09 \\
\hline Cerebrovascular disease & 23.0 & 24.9 & 22.4 & 24.6 & 0.05 & 0.84 \\
\hline COPD & 56.6 & 50.7 & 58.0 & 52.6 & $<0.001$ & 0.28 \\
\hline Liver disease & 1.4 & 1.3 & 1.3 & 1.5 & 0.95 & 0.72 \\
\hline CKD & 44.0 & 43.2 & 46.1 & 34.8 & 0.05 & $<0.001$ \\
\hline Diabetes & 38.7 & 41.6 & 38.7 & 37.4 & 0.05 & 0.01 \\
\hline Neoplastic disease & 29.0 & 35.8 & 28.0 & 30.4 & $<0.001$ & 0.001 \\
\hline HIV/AIDS & 0.3 & 0.3 & 0.3 & 0.4 & 0.56 & 0.86 \\
\hline Systemic corticosteroids & 29.6 & 26.5 & 30.5 & 26.5 & 0.003 & 0.98 \\
\hline Pulmonary medications & 41.9 & 35.6 & 43.6 & 37.1 & $<0.001$ & 0.38 \\
\hline Noninvasive mechanical ventilation & 1.2 & 2.0 & 1.1 & 1.0 & 0.01 & 0.01 \\
\hline \multicolumn{7}{|l|}{ Organ failure } \\
\hline Any organ failure & 18.0 & 22.0 & 17.9 & 16.4 & 0.001 & $<0.001$ \\
\hline Neurological & 1.6 & 1.7 & 1.5 & 2.0 & 0.77 & 0.52 \\
\hline Renal & 17.6 & 21.9 & 17.6 & 15.7 & 0.0002 & $<0.001$ \\
\hline Haematological & 2.3 & 2.6 & 2.2 & 2.7 & 0.29 & 0.99 \\
\hline Hepatic & 0.2 & 0.2 & 0.2 & 0.2 & 0.74 & 0.72 \\
\hline
\end{tabular}

Data are presented as median (interquartile range) or \%, unless otherwise stated. Bold indicates statistical significance. GC: guideline-concordant; HCAP: healthcareassociated pneumonia; CAP: community-acquired pneumonia; COPD: chronic obstructive pulmonary disease; CKD: chronic kidney disease. 
higher for GC-HCAP versus non-GC patients (median (IQR) 4 (2-6) versus $3(2-5) ; p<0.001)$. At baseline, GC-CAP patients had a higher prevalence of heart failure, COPD, tobacco use and prescriptions for cardiovascular medications, inhaled corticosteroids and pulmonary medications. GC-HCAP patients were more likely to have neoplastic disease. Organ failure did not differ significantly between GC-HCAP and GC-CAP patients.

\section{HCAP risk factors}

Overall, hospitalisation in the previous 90 days was the most common HCAP risk factor (table 2). Compared with GC-CAP patients, GC-HCAP patients were more likely to have a recent hospitalisation $(77.7 \%$ versus $62.4 \% ; \mathrm{p}<0.001)$ and present with multiple HCAP risk factors $(31.1 \%$ versus $21.0 \%$; $<<0.001$ ). There were no differences between GC-HCAP and GC-CAP patients regarding nursing home residence in the previous 90 days, haemodialysis or outpatient intravenous antibiotic therapy in the previous 90 days.

\section{GC antibiotic therapy}

Most patients $(83.7 \%)$ received antibiotic therapy concordant with either CAP or HCAP guidelines within $48 \mathrm{~h}$ of hospital admission. The most common GC-HCAP antibiotic regimen included an antipseudomonal $\beta$-lactam, an antipseudomonal fluoroquinolone, and either vancomycin or linezolid (82.1\%). Most other GC-HCAP patients received a similar regimen including an antipseudomonal $\beta$-lactam, an aminoglycoside and an MRSA-active agent (21.7\%). Patients receiving both an aminoglycoside and an antipseudomonal fluoroquinolone accounted for the overlap between the groups.
In GC-CAP patients, a respiratory fluoroquinolone was the most common regimen $(67.1 \%)$, followed by $\beta$-lactam plus macrolide $(45.0 \%)$. A significant number of patients prescribed GC-CAP antibiotics received both a respiratory fluoroquinolone and a $\beta$-lactam plus a macrolide (15.5\%). Nearly twothirds $(62.8 \%)$ of GC-CAP regimens included levofloxacin.

Non-GC patients received antibiotic regimens that did not meet minimum criteria for either CAP or HCAP guideline concordance. Many of these patients received insufficient HCAP coverage, with single antipseudomonal coverage present in $47.8 \%$ and double antipseudomonal coverage present in $13.5 \%$. One-third (30.9\%) received either vancomycin or linezolid, and $25.2 \%$ received a combination of an MRSA-active agent and one antipseudomonal agent.

\section{Bacterial pathogens}

Microorganisms were identified in $9.2 \%$ of patients (table 3 ). In patients with a positive culture, the most commonly isolated pathogens were S. pneumoniae $(27.8 \%)$, S. aureus $(26.0 \%)$, Pseudomonas (14.4\%) and Haemophilus influenzae (6.8\%). Atypical organisms and anaerobes were rare $(<3 \%)$.

Pseudomonas and S. aureus were identified more frequently in patients who received GC-HCAP versus GC-CAP therapy $(24.4 \%$ versus $10.5 \%, \mathrm{p}<0.001$; and $38.7 \%$ versus $16.7 \%$, $\mathrm{p}<0.001$, respectively). Compared with GC-HCAP patients, GC-CAP patients were more likely to have a positive culture for S. pneumoniae $(36.5 \%$ versus $14.2 \%, \mathrm{p}<0.001)$ or $H$. influenzae $(9.3 \%$ versus $0.9 \%, \mathrm{p}<0.001)$. In non-GC patients, S. aureus and

TABLE 3 Bacterial pathogen distribution

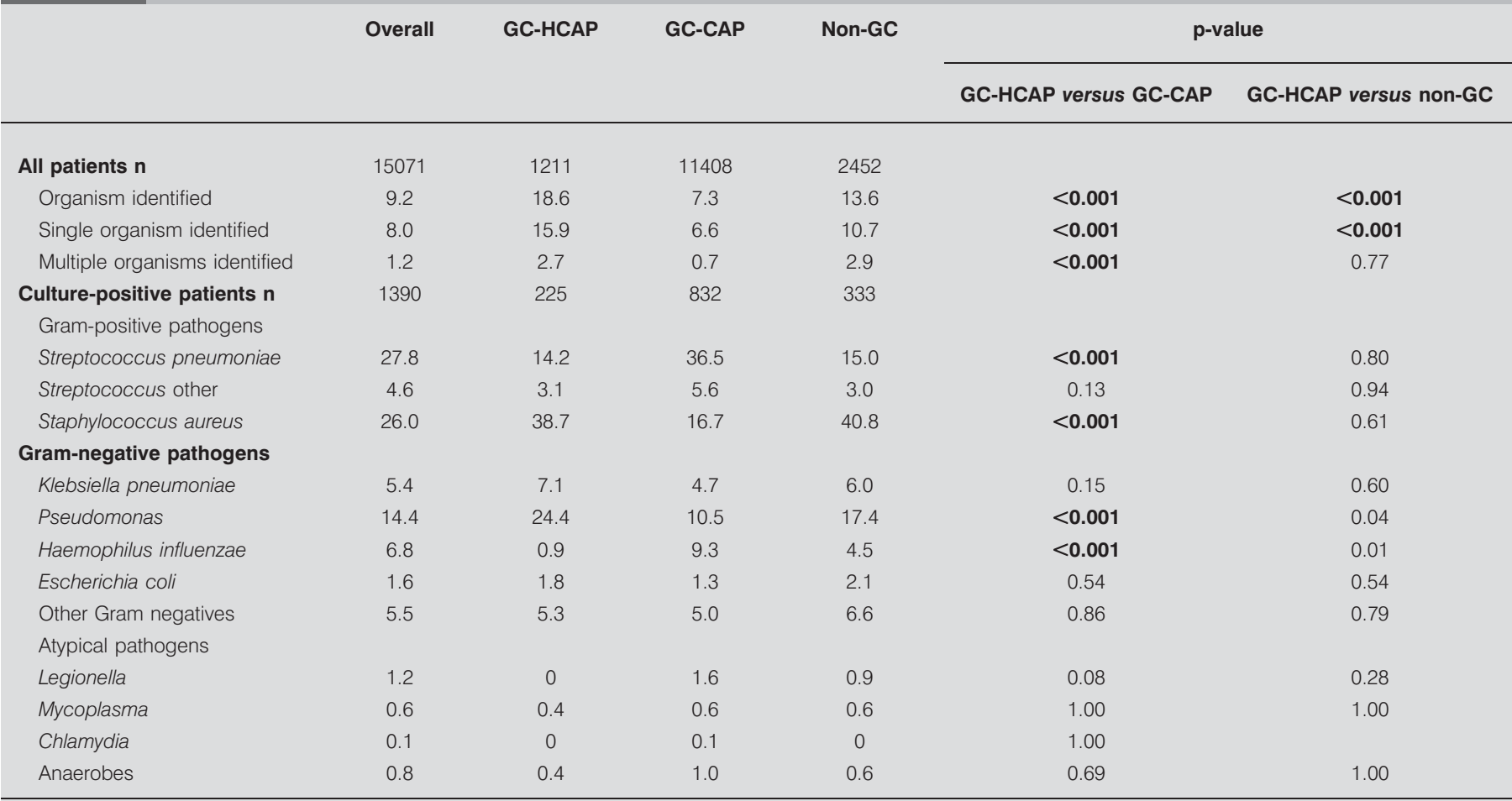

Data are presented as \%, unless otherwise stated. Bold indicates statistical significance. GC: guideline-concordant; HCAP: healthcare-associated pneumonia; CAP community-acquired pneumonia. 
Pseudomonas were the most frequently isolated pathogens $(40.8 \%$ and $17.4 \%$, respectively). GC-HCAP and non-GC patients were similar, without any statistically significant differences in bacterial pathogens.

\section{Health outcomes}

Median hospital LOS was 5 days (IQR 3-8 days) with 30-day and 90 -day mortality rates of $12.6 \%$ and $23.3 \%$, respectively (table 4). Compared with GC-CAP patients, GC-HCAP patients had a longer hospital LOS (median (IQR) 7 (4-13) versus 4 (37) days, $\mathrm{p}<0.001)$ and experienced higher rates of 30-day and 90day mortality $(22.8 \%$ versus $9.9 \%, \mathrm{p}<0.001 ; 37.8 \%$ versus $19.8 \%$, $\mathrm{p}<0.001$, respectively). There were no significant differences in 30- or 90-day mortality between GC-HCAP and non-GC patients; however, non-GC patients experienced shorter hospital LOS. Differences in mortality and hospital LOS remained the same when immunosuppressed (HIV/AIDS) patients were excluded from analyses.

Multilevel regression analysis of GC-HCAP and GC-CAP patients revealed several variables that were independently associated with 30-day mortality (table 5). The strongest independent predictors of 30-day mortality were hospital admission in the previous 90 days and GC-HCAP therapy (OR $(95 \%$ CI) 2.49 (2.12-2.94) and 2.18 (1.86-2.55), respectively). Other independent risk factors for 30-day mortality included cerebrovascular disease, neoplastic disease, noninvasive mechanical ventilation, neurological failure, renal failure and haematological failure. Tobacco use, recent prescription for cardiovascular medications and recent prescription for inhaled corticosteroids were protective.

When a propensity score for receipt of GC-HCAP therapy was calculated and entered into a second multivariable logistic regression model, GC-HCAP therapy continued to be an independent risk factor for 30-day mortality (OR 2.12, 95\% CI 1.82-2.48).

\section{Key pathogens and health outcomes by number of HCAP risk factors}

As the number of HCAP risk factors increased in an individual patient, changes were observed in pathogens and mortality (figs 2 and 3, respectively). Of particular interest, rates of pneumonia due to S. aureus and Pseudomonas increased as the number of HCAP risk factors increased from 1 to $>2$ (S. aureus $23.3 \%$ to $33.3 \%, \mathrm{p}<0.001$; Pseudomonas $14.0 \%$ to $21.4 \%, \mathrm{p}=0.39$ ). Patient mortality followed a similar pattern. From 1 to $>2$ risk factors, 30-day mortality increased from $11.6 \%$ to $17.2 \%$ $(p<0.001)$ and 90-day mortality increased from $21.9 \%$ to $29.1 \%(\mathrm{p}<0.001)$. Median hospital LOS was unchanged by the number of HCAP risk factors per patient.

\section{DISCUSSION}

The present study compared the effect of GC-HCAP and GCCAP therapy on health outcomes of patients with HCAP. In this national cohort of noncritically ill HCAP patients, GCHCAP therapy did not result in decreased patient mortality or hospital LOS.

Multiple prior studies have characterised the HCAP population. HCAP patients are older, present with more severe disease, suffer worse health outcomes and, as suggested by limited data from the USA, may be more likely to present with MDR pathogens [2-6]. Table 6 summarises the variation in selected HCAP pathogens among published data. Many similarities between HCAP patients in previous studies and HCAP patients in the present study were observed; however, mortality rates and hospital LOS were lower for HCAP patients in this study compared with previous research. This possibly reflects the exclusion of critically ill patients, which may have resulted in less severe disease and improved outcomes compared with cohorts including critically ill patients [9].

A limited number of HCAP studies have associated initial inappropriate antimicrobial therapy with increased mortality $[4,5,20]$. Two of these studies, one specific only to HCAP patients, determined that initial inappropriate therapy is an independent risk factor for in-hospital mortality [4, 20]. In contrast, RELLO et al. [21] recently evaluated HCAP and CAP patients with bacteraemic pneumococcal pneumonia and demonstrated that, despite low rates of inappropriate antibiotic therapy, mortality rates remained significantly higher in HCAP patients. Higher mortality in an HCAP cohort with low rates of inappropriate therapy alludes to potential fundamental differences between patients with CAP and HCAP.

Current guidelines recommend that HCAP patients should be treated with antibiotics similar to those used in nosocomial pneumonia; however, there is currently no evidence to demonstrate that GC-HCAP antibiotics will improve the survival rates of HCAP patients. In 2009, EL SOLH et al. [22] studied non-ICU pneumonia patients admitted to the hospital from nursing homes to compare differences in outcomes for

\section{TABLE 4 Health outcomes}

$$
\text { Overall GC-HCAP GC-CAP Non-GC p-value }
$$

GC-HCAP versus GC-CAP GC-HCAP versus non-GC

$\begin{array}{lcccccc}\text { Patients n } & 15071 & 1211 & 11408 & 2452 & & <0.001 \\ \text { Length of stay days } & 5(3-8) & 7(4-13) & 4(3-7) & 5(3-9) & <0.001 & 0.06 \\ \text { 30-day mortality } & 12.6 & 22.8 & 9.9 & 20.1 & <0.001 & 0.002\end{array}$

Data are presented as median (interquartile range) or \%, unless otherwise stated. Bold indicates statistical significance. GC: guideline-concordant; HCAP: healthcareassociated pneumonia; CAP: community-acquired pneumonia. 


\begin{tabular}{|c|c|c|c|}
\hline \multirow{2}{*}{$\begin{array}{l}\text { TABLE } 5 \\
\text { Risk factors }\end{array}$} & \multicolumn{3}{|c|}{$\begin{array}{l}\text { Risk factors for } 30 \text {-day mortality in guideline- } \\
\text { concordant healthcare-associated and } \\
\text { community-acquired pneumonia patients }\end{array}$} \\
\hline & & OR $(95 \% \mathrm{Cl})$ & p-value \\
\hline \multicolumn{2}{|l|}{ Sex } & $0.96(0.62-1.48)$ & 0.84 \\
\hline \multicolumn{2}{|l|}{ Race } & $1.11(0.99-1.24)$ & 0.08 \\
\hline \multicolumn{2}{|c|}{ Hispanic ethnicity } & $0.84(0.62-1.14)$ & 0.26 \\
\hline \multicolumn{4}{|c|}{ HCAP risk factors } \\
\hline \multicolumn{2}{|c|}{ Hospital admission within 90 days } & $2.49(2.12-2.94)$ & $<0.001$ \\
\hline \multicolumn{2}{|c|}{ Nursing home admission within 90 days } & $0.84(0.56-1.26)$ & 0.40 \\
\hline \multicolumn{2}{|c|}{ Haemodialysis } & $1.13(0.98-1.31)$ & 0.10 \\
\hline \multicolumn{2}{|c|}{ Outpatient i.v. antibiotics within 90 days } & $1.05(0.87-1.27)$ & 0.63 \\
\hline \multicolumn{4}{|c|}{ Comorbid conditions } \\
\hline \multicolumn{2}{|c|}{ Myocardial infarction } & $0.94(0.77-1.15)$ & 0.57 \\
\hline \multicolumn{2}{|c|}{ Heart failure } & $1.03(0.90-1.17)$ & 0.69 \\
\hline \multicolumn{2}{|c|}{ Cerebrovascular disease } & $1.20(1.05-1.37)$ & 0.01 \\
\hline \multicolumn{2}{|l|}{ COPD } & $0.92(0.80-1.06)$ & 0.28 \\
\hline \multicolumn{2}{|c|}{ Liver disease } & $1.02(0.62-1.68)$ & 0.94 \\
\hline \multicolumn{2}{|l|}{ Diabetes } & $0.85(0.72-1.01)$ & 0.07 \\
\hline \multicolumn{2}{|c|}{ Neoplastic disease } & $1.67(1.48-1.89)$ & $<0.001$ \\
\hline \multicolumn{2}{|l|}{ HIV/AIDS } & $1.12(0.39-3.28)$ & 0.83 \\
\hline \multicolumn{4}{|c|}{ Substance abuse or dependence } \\
\hline \multicolumn{2}{|c|}{ Tobacco use } & $0.73(0.64-0.83)$ & $<0.001$ \\
\hline \multicolumn{2}{|c|}{ Alcohol abuse } & $1.11(0.85-1.44)$ & 0.46 \\
\hline \multicolumn{4}{|c|}{ Medication use by class } \\
\hline \multicolumn{2}{|c|}{ Cardiovascular medications } & $0.67(0.58-0.76)$ & $<0.001$ \\
\hline \multicolumn{2}{|c|}{ Antidiabetic medications } & $0.89(0.73-1.08)$ & 0.23 \\
\hline \multicolumn{2}{|c|}{ Inhaled corticosteroids } & $0.70(0.59-0.82)$ & $<0.001$ \\
\hline \multicolumn{2}{|c|}{ Systemic corticosteroids } & $1.02(0.88-1.17)$ & 0.83 \\
\hline \multicolumn{2}{|c|}{ Pulmonary medications } & $1.04(0.89-1.20)$ & 0.65 \\
\hline \multicolumn{2}{|c|}{ Noninvasive mechanical ventilation } & $1.75(1.12-2.74)$ & 0.01 \\
\hline \multicolumn{4}{|c|}{ Organ failure } \\
\hline Neurologic & & $1.52(1.03-2.26)$ & 0.04 \\
\hline Renal & & $1.36(1.16-1.59)$ & $<0.001$ \\
\hline Haematolo & & $1.80(1.31-2.48)$ & $<0.001$ \\
\hline Hepatic & & $2.21(0.83-5.87)$ & 0.11 \\
\hline GC-HCAP ve & sus GC-CAP & $2.18(1.86-2.55)$ & $<0.001$ \\
\hline
\end{tabular}

Bold indicates statistical significance. HCAP: healthcare-associated pneumonia; COPD: chronic obstructive pulmonary disease; GC: guideline-concordant; CAP: community-acquired pneumonia. Variables were ordered to compare presence versus absence of characteristic when possible; patient sex and race were ordered as male versus female and black versus nonblack, respectively.

those treated with GC-HCAP therapy and GC-CAP therapy. No differences regarding in-hospital or 30-day mortality were found between the GC-CAP and GC-HCAP groups, and GCCAP patients actually had a decreased time to oral therapy and a decreased hospital LOS. Our analysis supports these notions regarding 30-day mortality and hospital LOS and, additionally, was not limited only to nursing home patients. In the present study, GC-HCAP therapy was not associated with improvements in patient LOS or mortality; in fact, GC-HCAP was associated with a longer LOS and increased mortality rates.

GC-HCAP and non-GC patients were mostly similar in baseline characteristics, pathogens and mortality outcomes; however, non-GC patients experienced a 2-day decrease in

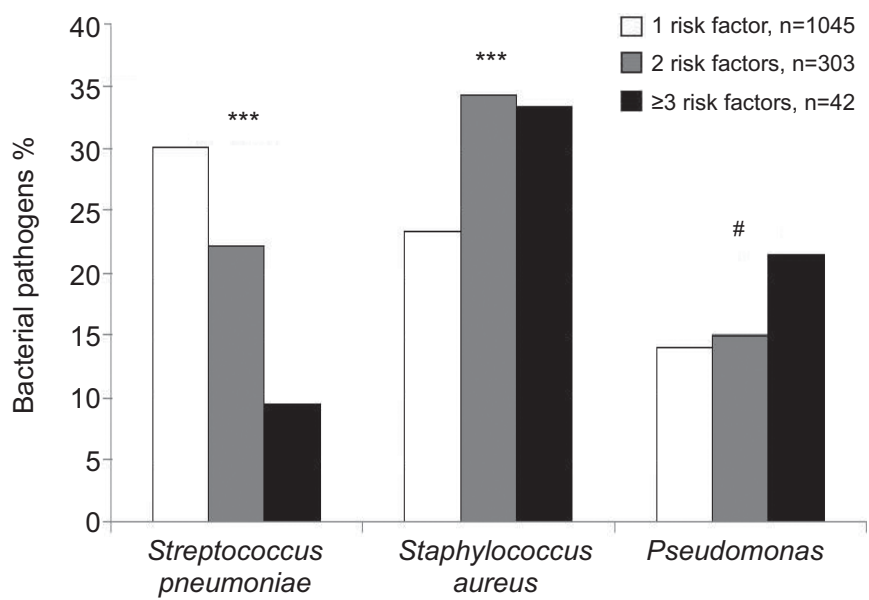

FIGURE 2. Bacterial pathogens in culture-positive healthcare-associated pneumonia (HCAP) patients, by number of HCAP risk factors. $n=1,390{ }^{*}: p=0.39$; $\star * *: p<0.001$.

LOS. We attribute this to the fact that GC-HCAP regimens may be more complex to manage and not as easily transferable to oral antibiotic therapy, similar to the GC-CAP population in the aforementioned study by EL SOLH et al. [22].

Despite great variation in HCAP pathogens throughout the published HCAP literature, HCAP patients consistently suffer at least double the mortality rate of CAP patients. Clinicians should be aware that these patients may be at an increased risk for poor outcomes; however, it is improper to mechanically prescribe broad-spectrum antibiotics to all patients with HCAP risk factors. Clinicians should be informed of their local epidemiology and use this information to afford a balance between appropriate empirical antibiotic therapy and overtreatment leading to resistance, increased adverse effects and increased costs.

Even with guidelines and identified risk factors, the selection of patients that need coverage for MRSA and Pseudomonas still requires some subjectivity and sound clinical judgment. It is noteworthy that prescribers in our study were relatively successful, as demonstrated by initial antimicrobial therapy,

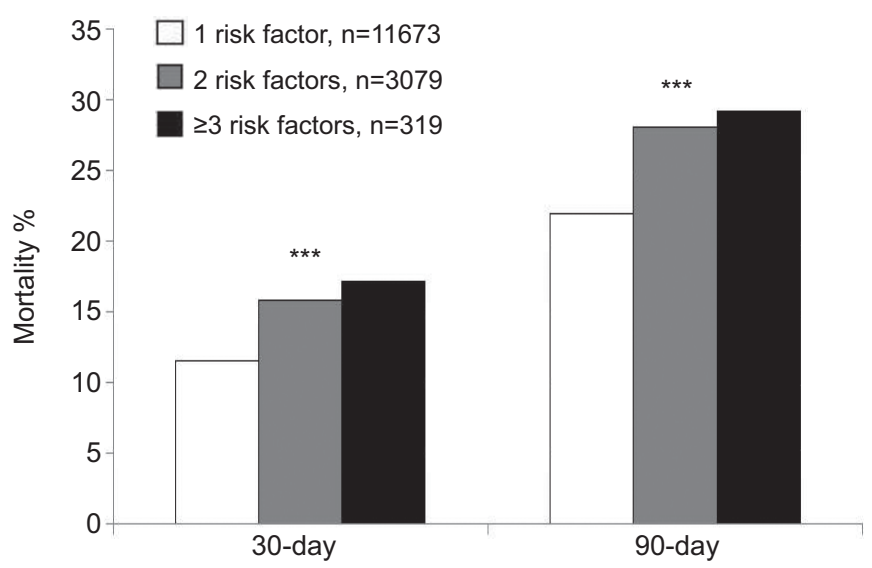

FIGURE 3. 30-day and 90-day mortality in healthcare-associated pneumonia (HCAP) patients, by number of HCAP risk factors. $n=15,071 .{ }^{* \star *}$ : $p<0.001$. 


\begin{tabular}{|c|c|c|c|c|c|}
\hline Streptococcus pneumoniae & 5.5 & 27.8 & 10.4 & 13.5 & 27.8 \\
\hline Staphylococcus aureus & 46.7 & 2.4 & 44.5 & 9.9 & 26.0 \\
\hline MRSA $^{\#}$ & 56.8 & 0 & 68.8 & 35.7 & \\
\hline
\end{tabular}

Data are presented as \%, unless otherwise stated. MRSA: methicillin-resistant Staphylococcus aureus. ${ }^{\#}$ : as a percentage of total S. aureus.

at predicting bacterial pneumonia pathogens. Information from prior treatment (prior antibiotics or pathogens) may have played a role in decisions, but this information was not available. While it is reassuring to see the results, we are unable to elicit the reason for these prescriber tendencies.

We believe it is unlikely that GC-HCAP therapy itself was responsible for the detriment in LOS and mortality. Rather, we believe confounding factors not captured in our study, including severity of disease and functional status, probably influenced these differences. Prognostic scoring systems validated in CAP patients, such as the Pneumonia Severity Index and CURB65 (which measures the risk factors confusion, urea nitrogen, respiratory rate, blood pressure and age $\geqslant 65 \mathrm{yrs}$ ), have demonstrated an increased mortality in patients with an increased severity of disease on admission $[23,24]$. Use of these methods, along with the included Charlson Index scores, may have helped to explain mortality differences between treatment groups. Additionally, poor functional status has proven to be a strong predictor of mortality and has been associated with an increased risk of resistant pathogens in pneumonia patients [25, 26]. A recent review of the concept of HCAP discusses the importance of functional status in pneumonia prognostication and suggests subgroups based on activities of daily living (ADL) scores may be useful in future classification systems [27].

It has also been proposed that physician- and/or patientdirected limitations on advanced care and aggressive intervention (e.g. ICU admission, invasive mechanical ventilation and/or vasopressor therapy) may be partially responsible for increased mortality in some HCAP patients [21, 27]. By excluding patients with critical illness, we were able potentially to limit the amount it contributed to increased mortality; however, it is possible that physician decision and family wishes to limit aggressive intervention in terminally ill patients could have affected mortality if these patients were not distributed equally between treatment groups.

Currently, two studies have evaluated the impact of individual HCAP risk factors on the risk of infection with a drug-resistant pathogen $[7,8]$. Each group of investigators identified multiple individual risk factors that were independent predictors of pneumonia due to a resistant pathogen. Residence in a nursing home or long-term care facility was the only predictor common to both studies. SCHREIBER et al. [8] also described an increased risk of resistant pathogens in patients with two or more HCAP risk factors compared with those with either one or zero risk factors. Similarly, we described the impact of cumulative
HCAP risk factors and found increased rates of $S$. aureus and Pseudomonas as patients possessed more HCAP criteria. Furthermore, we identified a positive correlation between the number of HCAP risk factors and patient mortality. Future research characterising the negative effects of cumulative HCAP criteria may help focus attention to the group of HCAP patients with the highest risk for poor outcomes.

While the current study provides valuable information among a national patient cohort, there are limitations. First, it was a retrospective cohort study in a predominantly elderly male population and is subject to the inherent limitations of all retrospective research. Multilevel regression techniques and propensity scores were used in an attempt to account for confounders and limit any potential biases; however, these methods were unable to fully account for all confounders and are not equivalent to the strengths of a prospective, randomised study.

Secondly, the use of ICD-9-CM codes to identify pneumonia patients, pathogens and baseline characteristics can be potentially problematic. This approach is common in large database studies and often necessary to enable efficient data collection. Many current HCAP studies are limited by single-centre or regional study sites and relatively small sample sizes. The use of ICD-9-CM codes enabled us to obtain significant amounts of information from a large national cohort of patients in a closed health system, a major strength of this study. The process of medical coding introduces several opportunities for human error and potential bias; however, data analysing ICD-9-CM codes for inpatient pneumonia patients have favourable positive and negative predictive values $(85.5 \%$ and $97.2 \%$, respectively), indicating a relatively low likelihood of misclassification [28].

Thirdly, the culture positivity rate in our study is relatively low. Culture positivity rates vary widely between pneumonia studies, and our data probably reflect a reliance on sputum cultures in our population of noncritically ill patients. Previous research demonstrates the difficulty of procuring good quality sputum samples with definitive results, as well as the lower culture positivity rate seen among noncritically ill patients [9, 29].

Fourthly, we were unable to compare differences in functional status between treatment groups. While functional status is not often measured in pneumonia studies, there is evidence that poor functional status can result in poor outcomes [25]. When possible, future HCAP studies should include functional status 
to further quantify the effects it may have on bacterial pathogens and outcomes.

Lastly, it would be useful to have more detailed data on antibiotic timing and bacterial susceptibility. Part of the inclusion criteria for our study was receipt of initial antibiotic therapy within $48 \mathrm{~h}$; however, we have no further data on antibiotic timing within that window. Additionally, no data were provided on bacterial susceptibility or rates of methicillin resistance in patients with positive cultures for $S$. aureus. MRSA is a pathogen of interest in HCAP cohorts, but ICD-9CM codes from 2002-2007 did not differentiate between methicillin-sensitive $S$. aureus and MRSA. Consequently, without bacterial susceptibilities, we were unable to identify and compare patients who were escalated to appropriate antibiotic therapy after receiving initial inappropriate therapy.

In conclusion, GC-HCAP antibiotic therapy was not associated with improved 30-day mortality in this cohort of noncritically ill VHA HCAP patients in the USA. Additional research is needed to fully understand reasons for mortality in HCAP patients and to determine interventions that improve survival.

\section{SUPPORT STATEMENT}

This project was supported by a grant from the National Institute of Nursing Research (Bethesda, MD, USA; grant R01NR010828). The content is solely the responsibility of the authors and does not necessarily represent the official views of the National Institute of Nursing Research or the National Institutes of Health. This material is the result of work supported with resources and the use of facilities at the South Texas Veterans Health Care System (San Antonio, TX, USA) and The University of Texas at Austin. E.M. Mortensen, the principal investigator, had full access to all of the data in the study and takes responsibility for the integrity of the data and the accuracy of the data analysis. C.R. Frei is supported by a National Institutes of Health Grant KL2 RR025766, salary support from The University of Texas at Austin College of Pharmacy, and research support from Ortho-McNeil Janssen (Titusville, NJ, USA). M.I. Restrepo is supported by a National Institutes of Health Grant K23HL096054, salary support by the South Texas Veterans Health Care System Audie L. Murphy Division and The University of Texas Health Science Center at San Antonio. The funding agencies had no role in conducting the study, or role in the preparation, review or approval of the manuscript. The views expressed in this article are those of the authors and do not necessarily represent the views of the Dept of Veterans Affairs (Washington, DC, USA).

\section{STATEMENT OF INTEREST}

Statements of interest for C.R. Frei, M.I. Restrepo and A. Anzueto can be found at www.erj.ersjournals.com/site/misc/statements.xhtml

\section{ACKNOWLEDGEMENTS}

The authors would like to thank K. Ryan-Hummel, a research assistant at The University of Texas at Austin (Austin, TX, USA), for her assistance in editing the final draft of the manuscript. This manuscript was presented as a poster at the Interscience Conference on Antimicrobial Agents and Chemotherapy, September 12-15, 2010, Boston, MA, USA.

\section{REFERENCES}

1 Guidelines for the management of adults with hospital-acquired, ventilator-associated, and healthcare-associated pneumonia. Am J Respir Crit Care Med 2005; 171: 388-416.
2 Kollef MH, Shorr A, Tabak YP, et al. Epidemiology and outcomes of health-care-associated pneumonia: results from a large US database of culture-positive pneumonia. Chest 2005; 128: 3854-3862.

3 Carratala J, Mykietiuk A, Fernandez-Sabe N, et al. Health careassociated pneumonia requiring hospital admission: epidemiology, antibiotic therapy, and clinical outcomes. Arch Intern Med 2007; 167: 1393-1399.

4 Micek ST, Kollef KE, Reichley RM, et al. Health care-associated pneumonia and community-acquired pneumonia: a single-center experience. Antimicrob Agents Chemother 2007; 51: 3568-3573.

5 Venditti M, Falcone M, Corrao S, et al. Outcomes of patients hospitalized with community-acquired, health care-associated, and hospital-acquired pneumonia. Ann Intern Med 2009; 150: 19-26.

6 Shindo Y, Sato S, Maruyama E, et al. Health-care-associated pneumonia among hospitalized patients in a Japanese community hospital. Chest 2009; 135: 633-640.

7 Shorr AF, Zilberberg MD, Micek ST, et al. Prediction of infection due to antibiotic-resistant bacteria by select risk factors for health care-associated pneumonia. Arch Intern Med 2008; 168: 2205-2210.

8 Schreiber MP, Chan CM, Shorr AF. Resistant pathogens in nonnosocomial pneumonia and respiratory failure: is it time to refine the definition of health-care-associated pneumonia? Chest 2010; 137: 1283-1288.

9 Restrepo MI, Mortensen EM, Velez JA, et al. A comparative study of community-acquired pneumonia patients admitted to the ward and the ICU. Chest 2008; 133: 610-617.

10 Charlson ME, Pompei P, Ales KL, et al. A new method of classifying prognostic comorbidity in longitudinal studies: development and validation. J Chronic Dis 1987; 40: 373-383.

11 Deyo RA, Cherkin DC, Ciol MA. Adapting a clinical comorbidity index for use with ICD-9-CM administrative databases. J Clin Epidemiol 1992; 45: 613-619.

12 Elixhauser A, Steiner C, Harris DR, et al. Comorbidity measures for use with administrative data. Med Care 1998; 36: 8-27.

13 Angus DC, Linde-Zwirble WT, Lidicker J, et al. Epidemiology of severe sepsis in the United States: analysis of incidence, outcome, and associated costs of care. Crit Care Med 2001; 29: 1303-1310.

14 Martin GS, Mannino DM, Eaton S, et al. The epidemiology of sepsis in the United States from 1979 through 2000. N Engl J Med 2003; 348: 1546-1554.

15 Mandell LA, Wunderink RG, Anzueto A, et al. Infectious Diseases Society of America/American Thoracic Society consensus guidelines on the management of community-acquired pneumonia in adults. Clin Infect Dis 2007; 44: S27-S72.

16 Mortensen EM, Coley CM, Singer DE, et al. Causes of death for patients with community-acquired pneumonia: results from the Pneumonia Patient Outcomes Research Team cohort study. Arch Intern Med 2002; 162: 1059-1064.

17 Sohn MW, Arnold N, Maynard C, et al. Accuracy and completeness of mortality data in the Department of Veterans Affairs. Popul Health Metr 2006; 4: 2.

18 Borzecki AM, Christiansen CL, Loveland S, et al. Trends in the inpatient quality indicators: the Veterans Health Administration experience. Med Care 48: 694-702.

19 Frei CR, Mortensen EM, Copeland LA, et al. Disparities of care for African-Americans and Caucasians with community-acquired pneumonia: a retrospective cohort study. BMC Health Serv Res 10: 143.

20 Zilberberg MD, Shorr AF, Micek ST, et al. Antimicrobial therapy escalation and hospital mortality among patients with health-careassociated pneumonia: a single-center experience. Chest 2008; 134: 963-968.

21 Rello J, Lujan M, Gallego M, et al. Why mortality is increased in health-care-associated pneumonia: lessons from pneumococcal bacteremic pneumonia. Chest 2010; 137: 1138-1144. 
22 El Solh AA, Akinnusi ME, Alfarah Z, et al. Effect of antibiotic guidelines on outcomes of hospitalized patients with nursing home-acquired pneumonia. J Am Geriatr Soc 2009; 57: 1030-1035.

23 Fine MJ, Auble TE, Yealy DM, et al. A prediction rule to identify low-risk patients with community-acquired pneumonia. $N$ Engl J Med 1997; 336: 243-250.

24 Lim WS, van der Eerden MM, Laing R, et al. Defining community acquired pneumonia severity on presentation to hospital: an international derivation and validation study. Thorax 2003; 58: 377-382.

25 Marrie TJ, Wu L. Factors influencing in-hospital mortality in community-acquired pneumonia: a prospective study of patients not initially admitted to the ICU. Chest 2005; 127: 1260-1270.
26 El Solh AA, Pietrantoni C, Bhat A, et al. Indicators of potentially drug-resistant bacteria in severe nursing home-acquired pneumonia. Clin Infect Dis 2004; 39: 474-480.

27 Ewig S, Welte T, Chastre J, et al. Rethinking the concepts of community-acquired and health-care-associated pneumonia. Lancet Infect Dis 2010; 10: 279-287.

28 Aronsky D, Haug PJ, Lagor C, et al. Accuracy of administrative data for identifying patients with pneumonia. Am J Med Qual 2005; 20: 319-328.

29 Garcia-Vazquez E, Marcos MA, Mensa J, et al. Assessment of the usefulness of sputum culture for diagnosis of communityacquired pneumonia using the PORT predictive scoring system. Arch Intern Med 2004; 164: 1807-1811. 